\title{
Lust statt Frust beim Reden über Alkohol
}

\author{
Regula Hälga, Stefan Neuner-Jehle ${ }^{b}$
}

${ }^{a}$ Infodrog; ${ }^{b}$ Kollegium für Hausarztmedizin

\begin{abstract}
Übermässiger Alkoholkonsum verursacht viel Leid und hohe Kosten. Je früher Alkoholprobleme erkannt werden, umso einfacher kann süchtig machendes Verhalten verändert werden. Ärztinnen und Ärzte leisten einen wichtigen Beitrag zur Früherkennung, wenn sie ihre Patientinnen und Patienten auf den Alkoholkonsum ansprechen. Doch das will gelernt sein!
\end{abstract}

Alkoholmissbrauch macht krank: Es gibt kaum ein menschliches Organ, das durch Alkohol nicht geschädigt werden kann. Leber und die Verdauungsorgane werden am stärksten beeinträchtigt. Jeder 12. Todesfall in der Schweiz ist auf Alkoholkonsum zurückzuführen, dies waren z.B. im Jahr 2011 rund 1600 Tote im Alter von 15 bis 74 Jahren. Drei von fünf dieser Todesfälle sind bedingt durch chronisch starken Alkoholkonsum [1]. Alkoholmissbrauch verursacht zudem jährliche Kosten von rund 4,2 Mrd. Franken [2].

Gleichzeitig befürworten 75 Prozent der Bevölkerung in der Schweiz Anreize für gesundheitsförderndes Verhalten [3]. Auch ist es für die Patienten in Ordnung, wenn sie von ihrem Arzt auf den Alkoholkonsum angesprochen werden, insbesondere wenn dies mit Wohlbefinden und Gesundheit begründet wird [4]. Aber was braucht es, damit das Gespräch über Alkohol in der Arztpraxis nicht zum Frust wird? Lust am Ausprobieren und motivierte Ärztinnen und Ärzte, die sich ganz einfach für den Alkoholkonsum ihrer Patientinnen und Patienten interessieren und gemeinsam mit ihnen über dessen Folgen für den Alltag und die Gesundheit nachdenken.

\section{Früherkennung}

Der Konsum von Alkohol bedeutet für viele Menschen Genuss. Dagegen ist grundsätzlich nichts einzuwenden. Aber wie viel ist zu viel? Die Eidgenössische Kommission für Alkoholfragen EKAL hat risikoarmen Konsum in ihrer Orientierungshilfe folgendermassen formuliert: Gesunde erwachsene Männer sollten nicht mehr als zwei bis maximal drei Gläser Alkoholisches pro Tag zu sich nehmen und Frauen nicht mehr als ein bis maximal zwei Gläser trinken. Zudem empfiehlt es sich, jede Woche an mehreren Tagen gar keinen Alkohol zu trinken. Wenn ausnahmsweise etwas mehr getrunken wird, dann sollten Männer nicht mehr als fünf und Frauen nicht mehr als vier Gläser konsumieren.

Je früher ein risikoreicher Alkoholkonsum erkannt wird, umso erfolgsversprechender ist es, das Konsum-

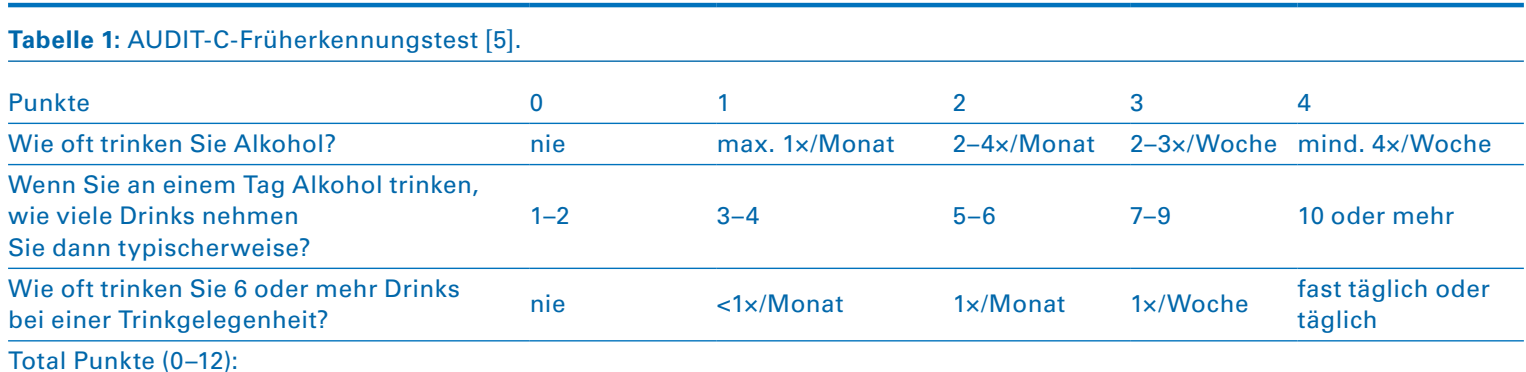

Bemerkung: 1 Drink entspricht $10 \mathrm{~g}$ Alkohol. 


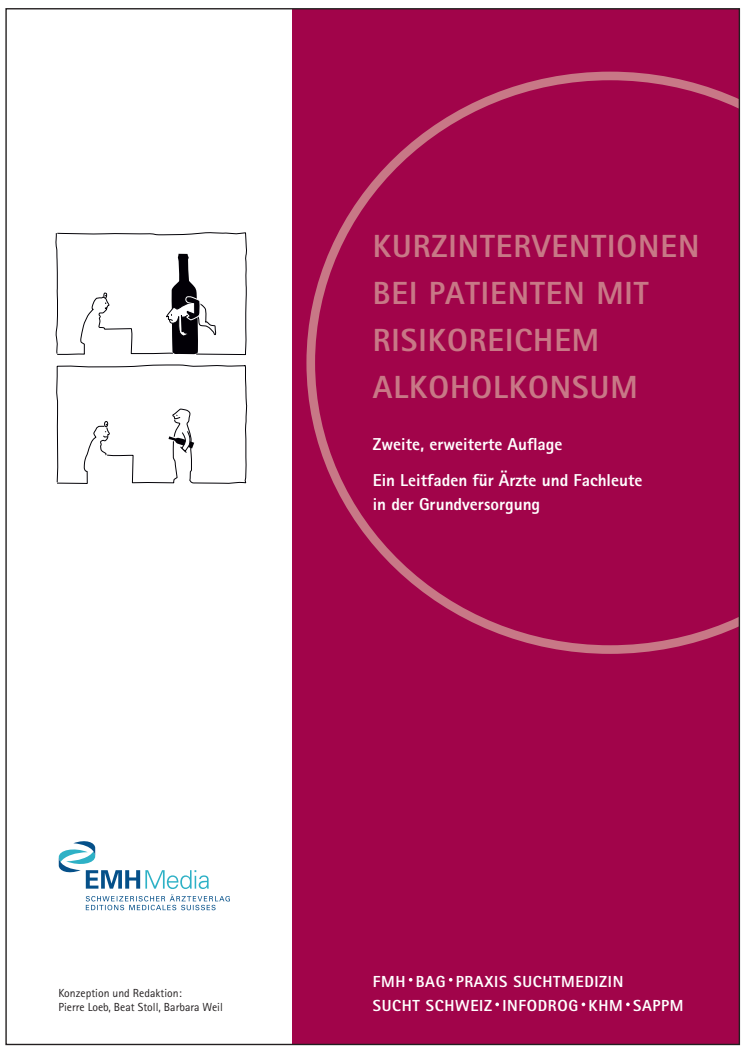

Abbildung 1: Die Broschüre Kurzinterventionen bei Patienten mit risikoreichem Alkoholkonsum [7]. Die Broschüre kann unter www.praxis-suchtmedizin.ch heruntergeladen oder im Sekretariat der Schweizerischen Akademie für Psychosomatische und Psychosoziale Medizin SAPPM per E-Mail für $5 \mathrm{CHF}$ (plus Porto und Verpackung) bestellt werden.

verhalten anzupassen resp. den Alkoholkonsum zu reduzieren!

\section{Ansprechen auf den Alkoholkonsum ...}

Aber wie mit den Patienten über gesundheitsförderliches Verhalten oder gar über den Alkoholkonsum ins Gespräch kommen? Ansprechen kann man die Patienten bei einer Routinekontrolle oder im Zusammenhang mit den Lebensgewohnheiten oder bei gesundheitlichen Beschwerden, welche durch einen risikoreichen Alkoholkonsum (mit-)verursacht sein können.

Die Haltung beim Ansprechen auf den Alkoholkonsum ist von zentraler Bedeutung. Der Patient ist Experte für seine Lebensweise und grundsätzlich motiviert für sein eigenes Wohl. Er wird also als Partner angesprochen, z.B.: «Darf ich Ihnen einige Fragen zum Alkoholkonsum stellen?» Wird die Frage negativ beantwortet, ist dies zu akzeptieren. Wichtig ist, zu zeigen, dass der Arzt jederzeit bereit ist, über das Thema Alkohol zu sprechen. Patienten begrüssen es in der
Regel, auf ihren Alkoholkonsum angesprochen zu werden.

Einfach und zuverlässig lässt sich mit den drei Fragen des AUDIT-C (Tab. 1) feststellen, ob ein risikoreicher Alkoholkonsum besteht [5]. Dem Patienten kann der AUDIT-Fragebogen auch abgegeben werden, mit der Möglichkeit, diesen bis zur nächsten Konsultation auszufüllen und zu besprechen. Oder er kann auf Online-Selbsttests hingewiesen werden; die elektronisch generierten Rückmeldungen werden in der Regel als weniger konfrontierend erlebt.

\section{... und intervenieren}

Wenn ein risikoreicher Alkoholkonsum vorliegt, gilt es, den Patienten sachlich über Risiken und mögliche Folgen zu informieren; moralisierende Informationen sind zu vermeiden. Es ist wichtig, dem Patienten die Möglichkeit zu geben, seinen eigenen Konsum zu hinterfragen, seinen persönlichen Fragen zuzuhören und sie zu beantworten. Bei einem risikoreichen Alkoholkonsum sind in der Hausarztpraxis Kurzinterventionen sehr geeignet. Sie sind in der medizinischen Grundversorgung gut erforscht und bewirken eine nachhaltige Konsumreduktion [6]. In der praktischen Anwendung sind Kurzinterventionen Gespräche von beschränkter Anzahl und kurzer Dauer. Sie orientieren sich am Konzept der Motivierenden Gesprächsführung und sind unterschiedlich stark strukturiert. Kurzinterventionen benötigen wenig Zeit - ein grosser Vorteil, ist der Zeitmangel bei Ärzten doch eine der grössten Barrieren bei der Umsetzung von ärztlichen Interventionen. Um Kurzinterventionen erfolgreich durchführen zu können, sind nebst dem Wissenserwerb das Einüben zentraler Fertigkeiten nötig. Die Broschüre Kurzinterventionen bei Patienten mit risikoreichem Alkoholkonsum [7] (Abb. 1) bietet Ärztinnen und Ärzten nicht nur Hintergrundwissen über Alkohol, sondern auch konkrete Hinweise zur Durchführung von Kurzinterventionen und Hilfestellungen beim Ansprechen.

\section{Informationen und Kampagne}

Es gibt zahlreiche Möglichkeiten, sowohl für Ärzte als auch für Patienten, sich vertiefter über Alkoholprobleme zu informieren, z.B. www.praxis-suchtmedizin.ch (v.a. für Ärzte und Fachpersonen), www.suchtschweiz.ch, www.safezone.ch

Die Alkoholpräventionskampagne des Bundesamts für Gesundheit und seiner Partner informiert im Online- und Papierquiz auf spielerische Weise über Risiken und Nebenwirkungen von Alkohol und ruft dazu auf, sich selber die Frage zu stellen, wie viel zu viel ist. Arztpraxen, Spitäler und weitere Interessierte können gratis den Quiz-Dispenser bestellen: www.alcohol-facts.ch 


\section{Motivierende Kurzintervention im Arzt-Patient-Gespräch}

Eine weitere Möglichkeit, das Ansprechen zu üben, sind Fortbildungen: Eine interdisziplinäre Arbeitsgemeinschaft - bestehend aus Forum Suchtmedizin Ostschweiz (FOSUMOS), Kollegium für Hausarztmedizin: Programm Gesundheitscoaching, Zürcher Fachstelle zur Prävention des Alkohol- und Medikamentenmissbrauchs (ZüFAM) und Zürcher Fachstelle für Alkoholprobleme (ZFA) - führt Sensibilisierungs-, Fortund Weiterbildungskurse [8] für Ärztinnen und Ärzte zu Motivierender Kurzintervention unter Einbezug der Alkoholthematik durch. Angeboten werden Kurzinputs (1-2 h), halbtägige Einsteigerkurse sowie Tagesseminare. Ziel der Veranstaltungen ist, dass Ärzte Wissen und Fertigkeiten erwerben, um ihre Patientinnen und Patienten für einen gesunden Lebensstil zu motivieren und sie bei Verhaltensänderungen wirkungsvoll zu unterstützen. Die eingeübten Fähigkeiten können nicht nur bei Alkoholproblemen, sondern auch bei einer Vielzahl weiterer gesundheitsrelevanter Verhaltensweisen eingesetzt werden.

\section{Weitere Projekte zur Förderung der Kurzintervention}

Infodrog, die Schweizerische Koordinations- und Fachstelle Sucht, und das Nationale Programm Alkohol finanzieren nicht nur die erwähnten Aktivitäten der interdisziplinären Arbeitsgemeinschaft mit, sondern auch das Pilotprojekt «Früherkennung und elektronische Kurzintervention bei problematischem Alkoholkonsum in der Arztpraxis» des Universitätsspitals Lausanne: In insgesamt acht Arztpraxen werden die wartenden Patientinnen und Patienten motiviert, auf einem Tablet Fragen zu ihrem Alkoholkonsum zu beantworten und das Resultat anschliessend mit dem Arzt zu besprechen. Erste Ergebnisse werden im Herbst 2016 erwartet.

\section{Fazit}

Eine frühzeitige Wahrnehmung und eine offene Kommunikation zum problematischen Alkoholkonsum ist eine wichtige und letztlich auch dankbare Aufgabe für Hausärzte. Ob diese Aufgabe eher frustrierend und schwierig oder aber «lustvoll», als Herausforderung wahrgenommen wird, hängt vor allem davon ab, ob es gelingt, den Patienten mit einer offenen, gleichberech-
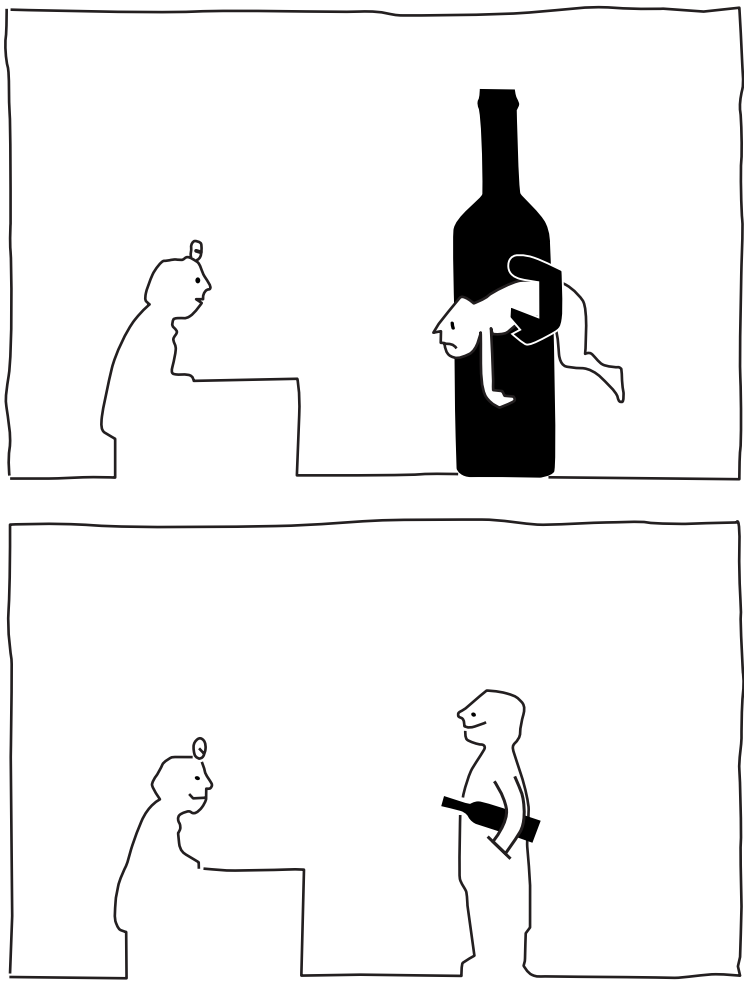

tigten Haltung zu begegnen, und ob man über ein Repertoire an kommunikativen Fertigkeiten verfügt (oder bereit ist, sich ein solches anzueignen).

\section{Literatur}

1 Marmet S, Gmel G, Gmel G, Frick H, Rehm J, Shield KDC. (2013). Alcohol-attributable mortality in Switzerland between 1997 and 2011. Lausanne: Sucht Schweiz.

2 Fischer B, Telser H, Widmer P, Leukert K. (2014). Alkoholbedingte Kosten in der Schweiz. Schlussbericht im Auftrag des Bundesamtes für Gesundheit. Olten: Polynomics.

3 pharma:ch 1/2008 Prävention: Investition in die Gesundheit und die Wohlfahrt eines Landes, vgl. http://www.interpharma.ch/ gesundheitswesen/1586-gesundheitsfoerderung-wird-immerwichtiger, Zugriff 20.7.2016.

4 Daeppen JB, Gaume MJ. (2006). Implémentation et dissémination de l'intervention brève pour la consommation d'alcool à risque en médecine de premier recours: Evaluation du projet partiel «médecins» du programme nationale alcool «Ça débouche sur quoi?». Schlussbericht im Auftrag des Bundesamtes für Gesundheit. Lausanne: CHUV.

5 Bush K, Kivlahan DR, McDonell MB. (1998). The AUDIT alcohol consumption questions (AUDIT-C): an effective brief screening test for problem drinking. Ambulatory Care Quality Improvement Project (ACQUIP). Alcohol Use Disorders Identification Test. Arch Intern Med;158:1789-95.

6 Bertholet N, Daeppen JB, Wietlisbach V, Fleming M, Burnand B. (2005). Reduction of Alcohol Consumption by Brief Alcohol Intervention in Primary Care - Systematic Review and Meta-analysis. Arch Intern Med. 2005; 165: 986-95.

7 Herausgeberschaft: FMH, BAG, Praxis Suchtmedizin, Sucht Schweiz, Infodrog, KHM und SAPPM, 2014.

8 Kursangebot: siehe unter www.zfa.ch/index.php/fortbildungaerzte.html 